\title{
Correlation of Fibroblast Activation Protein (FAP) immunohistochemical expression with histopathological grading and Tumor-Stroma Ratio (TSR) in colorectal adenocarcinoma
}

\author{
Sylvia Hilda, Betty, T. Ibnu Alferraly, Delyuzar, Soekimin
}

Email address: sylviahilda84@gmail.com

Department of Anatomical Pathology, Faculty of Medicine, Universitas Sumatera Utara, Medan, Indonesia

\begin{abstract}
Background: Colorectal adenocarcinoma is a malignancy of the large intestine (colon and rectum) of epithelial origin, ranks third in the world, and the second leading cause of cancer death. The prognosis can be assessed based on the reaction of the tumor microenvironment that plays a role in the stage of cancer development. Immunohistochemical examination of Fibroblast Activation Protein (FAP) as well as an assessment of grading and how the ratio between the extent of tumor and stroma (Tumor-Stroma Ratio) has recently been one of the prognostic predictors of colorectal adenocarcinoma.

Objective: To analyze the relationship of immunohistochemical expression of Fibroblast Activation Protein (FAP) with histopathological grading and Tumor-Stroma Ratio (TSR) in colorectal adenocarcinoma.

Materials and Methods: 32 samples in the form of histopathological slides from surgical tissue with a diagnosis of colorectal adenocarcinoma, reviewed slides and assessed for grading and TSR scores, then performed immunohistochemical staining of Fibroblast Activation Protein (FAP) and assessed the expression, then analyzed for correlation between variables using the Somers'd correlation test.

Results: The most histopathological grading distribution was low grade, the highest TSR was TSR-High, and the highest FAP expression found was strong expression. Based on the Somer's d correlation test, it was found that there was a significant correlation between FAP expression and grading $(\mathrm{p}=0.037)$ and there was no significant correlation between FAP expression and TSR $(\mathrm{p}=0.244)$.

Conclusion: The tumor microenvironment is one of the prognostic predictor factors in colorectal adenocarcinoma patients, should be reported in the routine pathology report and further investigated how the stromal component and the role of FAP expression to open the possibility of other targeted therapies.
\end{abstract}

Keywords : Fibroblast Activation Protein; Tumor-Stroma Ratio; colorectal prognosis.

\section{Introduction}

Colorectal carcinoma is defined as a malignant tumor of the large intestine (colon and rectum) originating from the epithelial component, with invasion of tumor cells between the stroma of the muscularis mucosal layer to the submucosa layer. Most colorectal malignancies (90\%) are adenocarcinomas. Colorectal cancer is one of the leading causes of morbidity and mortality in the world. The high incidence of colorectal carcinoma is associated with several risk factors, such as a diet high in calories and animal fat (Western type) accompanied by a lifestyle with low physical activity. The pathogenesis of colorectal adenocarcinoma is mostly preceded by a history of precursor lesions such as adenoma and dysplasia [1-4].

According to data from Colorectal Cancer Facts and Figures 2020-2022, in the United States in 2020 it is estimated that there will be 104,610 new cases of colon cancer and 43,340 new cases of rectal cancer with the total number of new cases of colorectal cancer is 147,950 and the death rate is around 53,200 cases. The majority of patients are aged 50 years and over, but cases are also found in individuals younger than 50 years, 
which is around 17,930 (12\%) new cases or the equivalent of 49 new cases per day [1,5] According to the 2018 Global Burden Cancer (GLOBOCAN), Asia ranks second-most for all types of cancer in both men and women, with the incidence rate of new cases of colonic carcinoma which ranks fifth of all cancers. The death rate from colon cancer ranks sixth of all cancer deaths, while rectal carcinoma itself ranks seventh most of all cancers and ranks eighth most of all cancer deaths [6]. In Indonesia, data obtained from GLOBOCAN in 2018, colorectal cancer ranks fourth after breast, cervical, and lung cancer, with an incidence of new cases of $30,017(8.6 \%)$. Based on gender, colorectal cancer in men ranks second after lung cancer with an incidence of new cases of $19,113(11.9 \%)$, and ranks fourth in women after breast, cervical and ovarian carcinomas with an incidence of new cases of 10,904 (5.8\%) [7].

Indicators of prognostic factors in colorectal adenocarcinoma are very diverse, one of which is histopathological grading. The grading is assessed based on the extent of the presentation of the gland formation which is divided into 2 criteria, namely low grade (well to moderately differentiated) and high grade (poorly differentiated) [2]. Another indicator that is being studied and continues to be studied is the role of the tumor microenvironment, one of which is the tumor-stromal compartment, which plays an important role in the initiation and progression of cancer, where the interaction between the stroma with malignant cells and non-malignant cells occurs at various stages of tumorigenesis[10]. The comparison between tumor and stroma, known as the Tumor-Stroma Ratio (TSR), is closely related to prognostic factors. The hypothesis obtained is that the wider the tumor stroma, the worse the patient's prognosis. TSR assessment can be done by simple examination using a light microscope and Hematoxylin \& Eosin (HE) staining [8,9].

Recently, in addition to colorectal carcinoma, studies to assess TSR have been carried out on various types of cancer such as breast, esophageal, non-small cell lung carcinoma (NSCLC), endometrial, hepatocellular carcinoma, nasopharyngeal carcinoma, cervical carcinoma, and some solid tumors. others that exhibit a strong prognostic parameter, are very easy to perform and can be performed in routine daily practice by a pathologist in diagnosis. Previous studies have found that the relationship between tumor and stroma plays an important role in carcinoma biology including growth, transformation, and progression. The relationship of the extent of the stroma to the tumor is often associated with grading, a high degree of malignancy and a poor prognosis [820].

In recent years, the close relationship between cancer and its microenvironment has become increasingly clear. The four main components of the tumor microenvironment that have been identified are the immune component known as the tumor immune microenvironment, the vascular component, the extracellular matrix component, and the stromal component which is still being studied which consists of non-immune cells of mesenchymal origin known as Mesenchymal Stem Cells Stroma. (MSCs) and cancer-associated fibroblasts known as Cancer-Associated Fibroblasts (CAFs) [21]. Strong experimental evidence has shown that CAFs, can promote tumorigenesis and development through a variety of mechanisms, including proliferation, angiogenesis, invasion, survival, and suppression of immunity. CAFs can originate from various cells such as resident fibroblasts, various differentiated precursor cells, dedifferentiated or transdifferentiated mature cells, or even from tumor cells themselves. Resident fibroblasts are located in the tissue of origin of the primary tumor and are the main tissue source of CAFs in the tumor. CAFs can be characterized by positive expression of $\alpha$-SMA, FAP- $\alpha$, FSP-1, VEGF, and cytokines such as IL-6 and IL-8. Research continues to be developed to determine marker immunohistochemical markers specific for CAFs in several organs [21-24].

Fibroblast Activation Protein (FAP) is a tumor-associated antigen that is a serine protease involved in extracellular matrix remodeling and is highly expressed in the reactive subset of stromal fibroblasts in human epithelial carcinoma (more than 90\%). Stromal cells expressing FAP can suppress tumor immune responses due to the production of large amounts of stromal cell-derived factor-1 (SDF-1). SDF-1 attracts regulatory T cells (CD4+ subtype ) into tumors. FAP has many roles in the process of neoangiogenesis, invasion, and metastasis, and is currently being explored as a target for cancer therapy. In the context of immunotherapy involving $\mathrm{T}$ cells in targeting cancer cells, agents targeting FAP expression on cells might enhance therapeutic 
efficacy against both tumor cell types in both solid and metastatic tumors [25].

FAP is a strong target for immunotherapy, so the targeting of preclinical and clinical studies of FAP has been initiated. FAP expression is currently a new prognostic marker and therapeutic target in several solid organ cancers [26]. Reports continue to accumulate evidence showing that FAP, which is an important marker for CAFs, plays a major role in the development of many types of tumors. FAP is expressed in reactive CAFs in stromal and granulation tissue to accelerate wound healing. In another study, it was stated that histologically, tumors with high stroma showed a tendency to increase FAP expression compared to tumors with low stroma. FAP was higher expressed in the invasive site than in the center of the tumor [27].

Several previous studies discussing this hypothesis did not provide consistent conclusions based on a cohort approach or exploratory evaluation and still compared the assessment of FAP in tumor cells and the stroma in relation to tumorigenesis activity. This study has analyzed the intensity and proportion of FAP expression by immunohistochemical examination. This study suggested that the expression of FAP in the stroma improves tumor prognosis and poor survival in some patients with solid tumor types, including colorectal cancer, but other studies on colorectal cancer did not get statistically significant results, both on the overall survival value, tumor cell invasion, lymph node involvement and distant metastases [26-29]. Therefore, further exploration is needed to validate the presence of Fibroblast Activation Protein (FAP) in relation to the activity of the stroma in influencing tumorigenesis, angiogenesis, and prognosis, and in the future, it can be used as an adequate treatment in colorectal cancer patients.

\section{Materials and Methods}

We examined 32 samples from cases of colorectal adenocarcinoma at H. Adam Malik General Hospital, Medan, who were diagnosed histopathologically with hematoxylin-eosin (HE) staining. This study was a descriptive cross-sectional study in which each sample in this study was observed once and only at one time at assessing the characteristics and expression of immunohistochemistry of FAP in colorectal adenocarcinoma in H. Adam Malik General Hospital. This research was approved by the Ethics Commission for Conducting Health Research Medical Faculty of North Sumatra University with no: 327/KEP/USU/2021. This study displays the characteristics of the sample in the form of age, gender, histopathological grading, Tumor-Stroma Ratio (TSR), and expression of FAP. The sample used is a sample that had the inclusion and exclusion criteria. Inclusion criteria were slide preparations and paraffin blocks from surgery which were diagnosed as adequate and representative histopathologically colorectal adenocarcinoma with Hematoxylin \& Eosin staining and had age and gender data. Exclusion criteria were in the form of paraffin blocks recorded on data storage devices that were damaged or thinned so that they could not be re-cutted, as well as slide preparations containing tumor and stromal masses that could not be assessed for TSR scoring, such as a lot of mucin content, necrotic mass, and lymphocyte inflammatory cells weight [8].

TSR was assessed based on the percentage of the extent of the tumor cell component and stromal component and categorized according to TSR-High (carcinoma component $>50 \%$ or stromal component $\leq 50 \%=$ Stroma Low) and TSR Low ( carcinoma component $\leq 50 \%$ or stromal component $>50 \%$ = Stroma High) [8]. Assessment of FAP immunohistochemical expression stained on the membrane or cytoplasm of stromal cells, and assessed by looking at the 5 (five) large fields (400x magnification) of the most invasive tumor sections using a CX21 microscope and taking the average number of the five fields of view. The proportion score of the area of the stained cells was divided into: $0=\leq 25 \%, 1=26 \%-50 \%, 2=$ $51 \%-75 \%, 3=>75 \%$. The staining intensity score was divided into: $0=$ negative (unstained), $1=$ weak positive, 2 moderate positive, $3=$ strong positive. The final result is by adding up the area proportion score and the intensity score and is interpreted as a weak expression if the total value is $<4$, a strong expression if the total value is $\geq 4[28]$. 

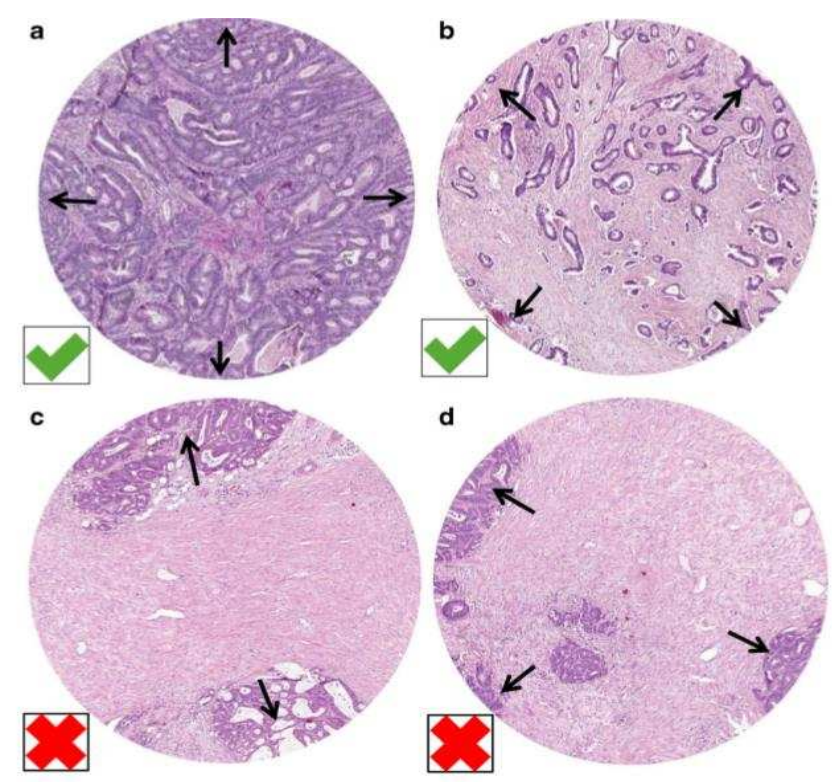

Fig 1. Example of assessing TSR in colorectal adenocarcinomas. a. stroma-low. b. stroma-high, with criteria for the presence of tumor vital cells in the four fields of view (arrow) that must be found and this is the correct scoring. c\&d. When tumor cells are only seen in two or three planes, this area cannot be assessed for TSR scoring. (Image using 100x magnification)[8].

\section{Result}

In this study, researchers took a minimum sample of 32 cases diagnosed histopathologically by conforming to the inclusion and exclusion criteria which aims to analyze the relation.nship of Fibroblast Activation Protein (FAP) immunohistochemistry expression with histopathological grading and TumorStroma Ratio (TSR) in colorectal adenocarcinoma. The following are the results of the research obtained. The sample in this study, based on clinical data from the medical records, showed that the youngest age was 28 years and the oldest age was 77 years. According to the age distribution of patients with colorectal carcinoma, the most cases were in the age group 51-60 years with 14 cases (43.8\%), followed by the age group 31-40 years, $41-50$ years, and 61-70 years, with the same number, each of 5 cases (15.6\%), 2 cases $(6.3 \%)$, and 1 case $(3.1 \%)$. Based on sex distribution, a male is the most common gender with colorectal adenocarcinoma which was found in 17 cases $(53.1 \%)$, while female sex was found in 15 cases $(46.9 \%)$. On microscopic examination, based on 2019 WHO histopathological grading, 11 cases of low grade (65.6\%) and high grade of 21 cases $(34.4 \%)$. The highest immunohistochemical expression of Fibroblast Activation Protein (FAP) was found in 19 cases (19\%), while the weak expression was found in 13 cases (40.6\%) (Table 1). 
Table 1. Frequency distribution of characteristics of colorectal adenocarcinoma

\begin{tabular}{lcc}
\hline \multicolumn{1}{c}{ Sample characteristics } & Total (n) & Percentage (\%) \\
\hline Age (years) & 1 & 3.1 \\
$21-30$ & 5 & 15.6 \\
$31-40$ & 5 & 15.6 \\
$41-50$ & 14 & 43.8 \\
$51-60$ & 5 & 15.6 \\
$61-70$ & 2 & 6.3 \\
$>70$ & & \\
Gender & 17 & 53.1 \\
Man & 15 & 46.9 \\
Woman & & 65.6 \\
Histopathological grading & 21 & 34.4 \\
Low grade & 11 & 65.6 \\
High grade & & 34.4 \\
Tumor-Stroma Ratio & 21 & 40.6 \\
High & 11 & 59.4 \\
$\quad$ Low & & $\mathbf{1 0 0}$ \\
$\quad$ Weak & 13 & \\
Strong & 19 & $\mathbf{3 2}$ \\
Total & & \\
\hline
\end{tabular}

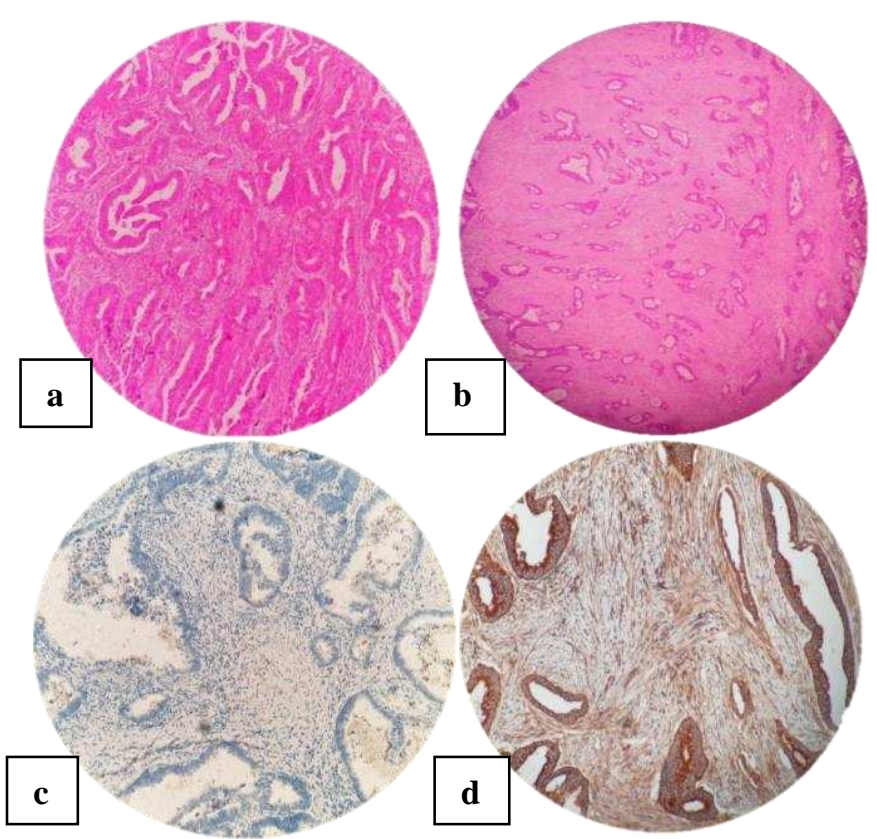

Fig 2. Microscopic examination. a. TSR-High. b TSR-Low. c. Weak FAP expression. d. Strong FAP expression. 
Based on the cross-tabulation table, weak expression of FAP was more common in colorectal adenocarcinoma with low grade in 11 cases (34.4\%) and in high grade in 2 cases (6.3\%). Strong expression of FAP was also more common in low grade colorectal adenocarcinoma in 10 cases (31.3\%) compared to a high grade in 9 cases $(28.1 \%)$.

Table 2. Characteristic distribution of the relationship between Fibroblast Activation Protein (FAP) immunohistochemical expression and histopathological grading of colorectal adenocarcinoma.

\begin{tabular}{lllllll}
\hline & \multicolumn{5}{c}{ Histopathological grading } & \\
No. & FAP expression & Low grade & High grade & Total \\
& & n & \% & n & \% & \\
\hline 1. & Weak & 11 & 34.4 & 2 & 6.3 & $13(40.6)$ \\
2. & Strong & 10 & 31.3 & 9 & 28.1 & $19(59.4)$ \\
& Total & 21 & 65.6 & 11 & 34.4 & $32(100)$ \\
\hline
\end{tabular}

Table 3. Correlation test of Fibroblast Activation Protein (FAP) immunohistochemical expression with histopathological grading of colorectal adenocarcinoma.

\begin{tabular}{|c|c|c|c|}
\hline \multirow{2}{*}{ Variable } & \multicolumn{3}{|c|}{ Histopathological grading } \\
\hline & $\mathbf{N}$ & Value(r) & $\mathbf{p}$ \\
\hline FAP expression & 32 & +0.320 & 0.037 \\
\hline
\end{tabular}

* Somers'd test because the data scale is ordinal-ordinal.

The results of the correlation test showed that the correlation between the immunohistochemical expression of Fibroblast Activation Protein (FAP) and the histopathological grading of colorectal adenocarcinoma had a significant correlation with $\mathrm{p}$-value $=0.037(\mathrm{p}<0.05)$. The Somers'd correlation coefficient shows a positive direction $(r$-value $=+0.320)$, where an increase in colorectal adenocarcinoma grading will increase the appearance of FAP expression, and vice versa.

Based on the immunohistochemical expression of FAP, weak expression of FAP was more common in colorectal adenocarcinoma with TSR-Low in 10 cases (31.3\%) and in TSR-High in 3 cases (9.4\%). Strong expression of FAP was found in TSR-Low in 11 cases (34.4\%) compared to TSR-High in 8 cases $(25.0 \%)$.

Table 4. Immunohistochemical expression of Fibroblast Activation Protein (FAP) with Tumor-Stroma Ratio (TSR) values in colorectal adenocarcinoma.

\begin{tabular}{lllllcc}
\hline & & \multicolumn{5}{c}{ TSR } \\
No. & FAP expression & \multicolumn{2}{c}{ High } & \multicolumn{2}{c}{ Low } & $\begin{array}{c}\text { Total } \\
\text { (\%) }\end{array}$ \\
& & $\mathbf{n}$ & $\boldsymbol{\%}$ & $\mathbf{n}$ & $\boldsymbol{\%}$ & \\
\hline 1. & Weak & 3 & 9.4 & 10 & 31.3 & $13(40.6)$ \\
2. & Strong & 8 & 25.0 & 11 & 34.4 & $19(59.4)$ \\
& Total & 11 & 34.4 & 21 & 65.6 & $32(100)$ \\
\hline
\end{tabular}

Table 5. Correlation test of FAP expression with TSR value in colorectal adenocarcinoma. 


\begin{tabular}{lllc}
\hline FAP expression & N & Value(r) & TSR \\
\hline TSR Category & 32 & -0.190 & 0.244 \\
\hline
\end{tabular}

*Somers'd Correlation Test due to ordinal-ordinal data scale.

The results of the correlation test showed that there was no significant correlation between FAP expression and TSR value with $p=0.244(p>0.05)$. Somers'd correlation coefficient shows the value category of $r=-0.190$, with a negative direction, where if there is an increase in the TSR value in colorectal adenocarcinoma, it will decrease FAP expression, and vice versa.

\section{Discussion}

Colorectal adenocarcinoma samples in this study were 32 samples recorded in the data storage software in the medical records of the Anatomic Pathology Unit RSUP H. Adam Malik Medan which met the inclusion and exclusion criteria. According to age distribution data, the highest age group in this study was 51-60 years old, with the youngest being 28 years old and the oldest being 77 years old. This is in line with the research conducted by Nasution at $\mathrm{H}$. Adam Malik Hospital in 2015-2017, the age of most patients found at the age above is the same as 50 years [30]. Based on the Colorectal Cancer facts and figures in 2020, most of them are above 65 years old, there were 60,780 new cases of colon carcinoma and 19,230 new cases of rectal carcinoma [1]. According to Arifputera, the risk factor for colorectal carcinoma, namely age, where the incidence increases after the age of 50 years. A person's chances of being diagnosed with colorectal cancer increase after the age of 50 years, more than $90 \%$ of colorectal cancer cases occur in patients over the age of 50 years. As people age, a person's risk of developing colorectal cancer increases. This can be caused by increased fat mass at that age and other risk factors that can be in the form of lack of physical activity, high consumption of fat, carcinogenic substances, consumption of red meat, alcohol, and smoking that are not balanced with fiber consumption and lack of initiative to carry out early detection that applies to both sexes [31]. Patients who were younger or under 50 years of age had higher five-year relative survival rates than those over 50 years of age [2]. According to the sex distribution, it was found that the male sex was found more than the female. In Nasution's study, data showed that the incidence of colorectal cancer was mostly found in males, namely 44 cases $(54.3 \%)$, while in women, namely 37 cases $(45.7 \%)$. Colorectal carcinoma also has a higher tendency to occur in men, about $4.4 \%$ of all men or 1 in 23 people will suffer from colorectal carcinoma, while in women, around $4.1 \%$ or 1 in 25 women will suffer from colorectal cancer. colorectal carcinoma during his lifetime [1].

Based on histopathological grading, low-grade colorectal adenocarcinoma ( $65.6 \%$ of cases) was found to be more common than high grade (34.4\% of cases). In previous studies that used 2010 WHO Digestive Systems criteria, the histopathological grading was divided into 3 categories: well differentiated, moderately differentiated, and poorly differentiated, while based on 2019 WHO Digestive Systems criteria, welldifferentiated and moderately differentiated were classified as low grade, and poorly differentiated classified into high grade [2,32]. The results of this study can be said to be the same as the results of research conducted by Nasution at H. Adam Malik Hospital Medan in 2015-2017, it was found that the most common grading of colorectal adenocarcinoma was well differentiated with $44.4 \%$ cases, followed by moderately differentiated as many as $35.8 \%$ cases and poorly differentiated $19.8 \%$ [30]. Similarly, research conducted by Hilda et al., which gets almost the same result [19]. Scheer et al., da Silva et al., and Mesker et al., grading colorectal adenocarcinoma is more commonly diagnosed at moderately differentiated $[10,33,34]$.

Grading is one of the various factors used to predict the growth and spread of tumor cells. In poorly differentiated (high grade), the growth and development of cancer cells will be faster than in well to moderately differentiated [35]. The higher the grade, the worse the prognosis. Five years survival rate for well 
differentiated is $99.3 \%$, moderately differentiated is $86 \%$, and poorly differentiated is $68 \%$ [36].

The assessment of Tumor-Stroma Ratio (TSR) has recently been widely studied to determine the prognosis in patients with colorectal adenocarcinoma. This assessment aims to observe the stromal compartment of the tumor, by observing the interaction of the tumor with the surrounding stroma which plays an important role in the initiation and progression of cancer. The assessment technique is very simple, so it can be applied in routine diagnostic practice by Anatomic Pathologists, by assessing the percentage ratio of the tumor and stromal components. In this study, the TSR-High score was found in 21 cases (65.6\%), more than the TSR-Low score which was only found in 11 cases $(34.4 \%)$. This is in accordance with previous research conducted by several Anatomic Pathologists, both junior and senior, who found that the TSR score was higher for TSR-High [34].

In one meta-analysis conducted to analyze the prognostic value of TSR in rectal cancer, it was found that a higher stromal component was significantly associated with several unfavorable prognostic factors such as histological grade, venous invasion, lymph node involvement status, and depth of invasion. tumor, so that it can predict the value of Disease Free Survival (DFS), Cancer-Specific Survival rates (CSS), and Overall Survival (OS) [37]. Malignant epithelial tumors from patients with poor prognosis show a high proportion of stroma, whereas tumors with extensive carcinoma tissue are associated with a good prognosis [917]. According to research conducted by Hansen et al., TSR values can also predict the recurrence of colon cancer patients treated with neoadjuvant chemotherapy [38].

Knowledge about tumor stroma has increased in recent years. Cancer-associated fibroblasts (CAFs) in the tumor stroma tend to influence tumor growth by altering the normal stromal microenvironment from initially acting as a tumor suppressor to being supportive of the tumor. However, the stromal mechanism in the development of rectal cancer is not completely clear. Tumor cells infiltrate the basement membrane and stimulate stromal cells to form a tumor microenvironment in the early stages of tumor invasion. Although the stromal cells are not malignant, tumor cells and surrounding stromal cells and other cells in the stroma will continue to interact, resulting in phenotypic and functional changes. Furthermore, these modifications lead to the recruitment of immune cells and endothelial cells, proteolysis, matrix remodeling, loss of cell adhesion, and cytoskeletal rearrangement, all of which are important processes in tumor development. Tumor-associated stromal components, including the extracellular matrix, various secreted factors, and several cell types. As an intermediary, the extracellular matrix allows cancer cells to communicate with stromal cells to enter the microenvironment and metastasize [39].

In tumor tissue, fibroblasts are the main cellular component of the tumor stroma which comprises an integral component of the tumor. In some types of cancer, fibroblasts make up a greater proportion of cells in the tumor than cancer cells. Fibroblasts in tumors have an activated phenotype known as Cancer-Associated Fibroblasts (CAFs). Several markers of CAFs have been studied, one of which is Fibroblast Activation Protein (FAP). In this study, FAP expression in 32 samples of colorectal adenocarcinoma was most commonly found with strong expression in 19 cases $(59.4 \%)$, while weak expression was found in 13 cases (40.6\%). FAP expression was associated with histopathological grading and Tumor-Stroma Ratio scores. Based on the results of the analysis using the Somers'd test, it was found that there was a significant correlation between FAP expression and histopathological grading with $\mathrm{r}$ value $=+0.320$ and $\mathrm{p}$ value $=0.037 \quad(\mathrm{p}<0.05)$. From the results of this analysis, it can also be concluded that an increase in colorectal adenocarcinoma grading will increase the appearance of FAP expression, and vice versa. In the study by Wikberg et al ., FAP expression had a significant relationship to grading, but the expression was higher in the tumor center area than the tumor front area. In this study, it was also found that the strong expression of FAP in the center of the tumor was more commonly found in right-sided tumors, while FAP expression in the front of the tumor was most commonly found in the rectal area. It was also found that only $10 \%$ of the samples did not show FAP expression in the tumor front. FAP expression was assessed in relation to tumor heterogeneity. Several previous studies have looked at FAP expression as essentially a prerequisite for invasion. It is thought that FAP expression by fibroblasts at the tumor front might reflect a 
stress response to the microtumor environment at the invasive margin [40]. In another study, it was found that FAP expression was only reported in the tumor stroma. FAP overexpression rates ranged between $54 \%$ and $93 \%$ in tumor stroma and between $50 \%$ and $100 \%$ expression in tumor cells. In theperitumoral compartment, expression of FAP mostly found in the CAF adjacent close to the tumor cells [41].

Histopathological grading should be based on the least differentiated component, assessing the percentage of glandular formation. Tumor grading is generally considered to be one of the independent prognostic variables, with high grade or poor differentiation associated with poorer survival [3]. Research by Yuan $\mathrm{Z}$ et al ., found that FAP was shown to promote migration and invasion due to the effects of cell proliferation [42]. According to a study by Errate et al ., FAP expression in primary colorectal carcinoma is also correlated with the grade, as well as sarcomatoid phenotype, which is thought to be associated with higher epithelial-tomesenchymal transition (EMT) activity [43].

The results of the FAP correlation test with the TSR score showed that there was no significant correlation between FAP expression and TSR value, $p=0.244$ ( $p>0.05)$. Somers'd correlation coefficient shows the value category of $r=-0.190$, with a negative direction where if there is an increase in the TSR value in colorectal adenocarcinoma, it will decrease FAP expression, and vice versa. From the research of Tessa et al. In 2019, it was found that FAP expression was higher in the invasive site than in the center of the tumor, with highstromal values [27].

CAFs have "various faces" in cancer development, this is because CAFs can inhibit cancer cells at an early stage utilizing tumor immunosurveillance, while at an advanced stage, CAFs will play a role in promoting cancer growth and development [21]. The precise tipping point between the function of CAFs in tissue repair and tumor promotion remains unclear $[44,45]$. One of the extracellular proteins or molecules that play a role in activating the mobilization of resident fibroblasts into CAFs is Transforming Growth Factor Beta 1 (TGF- $\beta 1$ ), which is secreted by the stroma and tumor cells. TGF- $\beta$ can induce tumorigenesis by inducing EMT. Under physiological conditions, EMT plays a role in the context of embryogenesis and tissue repair. This process can change and become pathological, and lead to the formation of fibrosis and tumorigenesis. In most carcinomas, progression to malignancy is followed by loss of epithelial differentiation/characteristics such as dissolution of tight junctions, adherens junctions, and desmosomes, loss of apical-basolateral polarity, and change to a mesenchymal phenotype characterized by actin reorganization, fiber stress formation, migration and transformation, invasion, known as EMT. Mesenchymal cells are associated with a poor prognosis, increased motility, and chemoresistance [46].

From the results of statistical analysis, although no significant results were obtained, there was a tendency that with the category value of $r=-0.190$, it can be concluded that these two variables have a negative direction, namely if there is an increase in the TSR value in colorectal adenocarcinoma, it will decrease FAP expression, and vice versa. In accordance with the literature, high stroma indicates high FAP expression, whereas higher stroma has a worse prognostic value. FAP is one of the markers for CAFs, which are the most abundant component in the tumor microenvironment. The tumor microenvironment can influence the growth and development of cancer cells. It has also been suggested that FAP overexpression in solid tumors is associated with lymph node metastasis and poor prognosis [26,27].

\section{Conclusion}

From 32 samples of adenocarcinoma in this study, the most cases were in the age group 51-60 years and male is the most common gender than female. Histopathological grading of colorectal adenocarcinoma with low grade $(65.6 \%)$ was more common than high grade $(34.4 \%)$, with Tumor-Stroma Ratio (TSR) High $(65.6 \%)$ was higher than TSR Low (34.4\%). Immunohistochemical expression of Fibroblast Activation Protein (FAP) in colorectal adenocarcinoma was more strongly expressed $(59.4 \%)$ than weak expression $(40.6 \%)$. The immunohistochemical expression of FAP has a significant correlation with the histopathological grading of colorectal adenocarcinoma, where the histopathological grading will increase 
the appearance of FAP expression, and vice versa. There is no significant correlation between the immunohistochemical expression of FAP and TSR, but there is a tendency if there is an increase in the TSR value, it will decrease the expression of FAP, and vice versa.

\section{Acknowledgements}

A big thanks to all teaching staff and residents of the Department of Pathology Anatomics, Universitas Sumatera Utara / H. Adam Malik Central General Hospital Medan for all your support and guidance.

\section{References}

1. American Cancer Society. Colorectal Cancer Facts \& Figures 2020-2022. Atlanta: American Cancer Society. 2020;pp.1-48.

2. Nagtegaal ID, Arends MJ, Salto-Tellez M. Colorectal adenocarcinoma. In WHO Classification of Tumours: Digestive System Tumours. $5^{\text {th }}$. WHO Classification of Tumours Editorial Board (Ed), International Agency for Research on Cancer. Lyon. 2019;p.177-87.

3. Fleming M, Ravula S, Tatishchev SF, Wang HL. Colorectal Carcinoma: Pathological Aspects. Journal of Gastrointestinal Oncology. 2012;3:pp.153-73.

4. Kumar V, Abbas AK, Fausto N, Aster JC. Oral Cavity and Gastrointestinal tract. Robbins Basic Pathology. 9 $^{\text {th }}$ ed. Philladelpia; Elsevier Saunders. 2013;pp.598-602.

5. Siegel RL, Miller KD, Jemal AJ. Cancer Statistic,2020. CA Cancer J Clin 2020. 2020;70:pp.7-30.

6. International Agency for Research on Cancer. Asia, Source : Globocan 2018. Available from : http:// co.iarc.fr/today/data/factsheets/populations/ 935-asia-fact-sheets.pdf.

7. International Agency for Research on Cancer. Indonesia, Source: Globocan 2018. Available from : http://gco.iarc.fr/today/data/factsheets/populations/360-indonesia-fact-sheets.pdf

8. van Pelt GW, Kjaer-Frifeldt S, van Krieken JHJM, Al Dieri, Morreau H, Tollenaar M, et al. Scoring the tumor-stroma ratio in colon cancer: procedure and recommendation. Virchows Archiv. 2018;473:pp.405-12.

9. Scheer R, Baidoshvii A, Zoidze S, Elferink MAG, Berkel AEM, Klaase JM, et al. Tumor-stroma ratio as prognostic factor for survival in rectal adenocarcinoma : A retrospective cohort study. World Journal of Gastrointestinal Oncology. 2017 December 15; 9(12):pp.466-74.

10. Zhao K, Li Z, Wang Y, Wu X, Xu Z, Wu L, et al. Artificial intelligence quantified tumour-stroma ratio is an independent predictor for overall survival in resectable colorectal cancer. EBioMedicine 61. 2020. pp.1-9.

11. Le NH, Franken P, Fodde R. Tumor stroma interactions in colorectal cancer: converging on $\beta$-catenin activation and cancer stemness. British Journal on Cancer. 2008;98:pp.1886-93.

12. Zhang T, Xu J, Shen H, Dong W, Ni Y, Du J. Tumor-stroma ratio is an independent predictor for survival in NSCLC. Int J Clin Exp Pathol 2015;8(9):pp.11348-55.

13. Panayiotou H, Orsi NM, Thygesen HH, Wright AI, Winder M, Hutson R, et al. The prognostic significance of tumour-stroma ratio in endometrial carcinoma. BMC Cancer. 2015;15:955:pp.1-8.

14. Wang K, Ma W, wang J, Yu L, Zhang X, Wang Z, et al. Tumor-stroma ratio is an independent predictor for survival in esophageal squamous cell carcinoma. J Thorac Oncol. 2012. pp.1457-61.

15. de Kruijf EM, van Nes JG, Van de V, Putter H, Smith VT, Liefers GJ, et al. Tumor-stroma ratio in the primary tumor is a prognostic factor in early breast cancer patients, especially in triple-negative carcinoma patients. Breast Cancer Res Treat. 2011;pp.687-96. 
16. Huijbers A, Tollenaar RA, van Pelt GW, Zeestraten EC, Dutton S, McConkey, et al. The proportion of tumor-stroma as a strong prognostic factor for stage II and III colon cancer patients: validation in the VICTOR trial. Ann Oncol. 2013;pp.179-85. Available from : https://academic.oup.com/annonc/article/24/1/179/1746464

17. Z Lv, X Cai, X Weng, H Xiao, C Du, J Cheng, et al. Tumor-stroma ratio is a prognostic factor for survival in hepatocellular carcinoma patients after liver resection or transplantation. Surgery. 2015;pp142-50.

18. J Liu, J Liu, Li J, Y Chen, $\mathrm{X}$ Guan, $\mathrm{X} \mathrm{Wu}$, et al. Tumor-stroma ratio is an independent predictor for survival in early cervical carcinoma. Gynecol Oncol. 2014;pp.81-6. Available from : https://doi.org/10.1016/j.ygyno.2013.11.003

19. Hilda S, Soekimin, Betty. Correlation of Tumor-Stroma Ratio (TSR) with histological grading and staging in colorectal adenocarcinoma. International Journal of Research Publication. 2020;45:1:pp.1-9. [cited 2020 jan 27].

20. Purba FO, Soekimin, Alferraly TI. Correlation of Tumor-Stroma Ratio (TSR) with primary tumor size and lymph node involvement in nasopharyngeal carcinoma. International Journal of Research Publication. 2019;pp.1-6. [cited 2021 jan 27].

21. Louault K, Li R, DeClerck YA. Cancer-Associated Fibroblast: Understanding Their Heterogenity. Cancers 2020; 12:3108:pp.1-28.

22. Hui L, Chen Y. Tumor Microenvironment: Sanctuary of the Devil. Cancer Lett. 2015;pp.7-13.

23. Yang F, Wang T, Du P, Fan H, Dong X, Guo H. M2 Bone Marrow-Derived Macrophage-Derived Exosomes Shuffle MicroRNA-21 to Accelerate Immune Escape of Glioma by Modulating PEG3. Cancer Cell Int. 2020;20:pp1-7.

24. Gajewski, TF, Schreiber H, Fu Y. Innate and Adaptive Immune Cells in the Tumor Microenvironment. Nat. Immunol. 2013;pp.1014-22.

25. Jiang GM, Xu W, Du J, Zhang KS, Zhang QG, Wang XW, et al. The application of the fibroblast activation protein $\alpha$-targeted immunotherapy strategy. Oncotarget. 2016;7(22):pp.33472-82.

26. Liu F, Qi L, Liu B, Liu J, Zhang H, Che D, et al. Fibroblast Activation Protein Overexpression and Clinical Implications in Solid Tumors: A Meta-Analysis. PLOS One. 2015;10(3):pp.1-18.

27. Sanderg TP, Stuart MPME, Oosting J, Tollenaar RAEM, Sier CFM, Mesker WE. Increased expression of cancer-associated fibroblast markers at the invasive front and its association with tumor-stroma ratio in colorectal cancer. BMC Cancer. 2019;19(284):pp.1-9.

28. Cao F, Wang S, Wang H, Tang H. Fibroblast activation protein- $\alpha$ in tumor cells promotes colorectal cancer angiogenesis via the Akt and ERK signaling pathways. Molecular Medicine Reports 17.2018;pp.2593-99.

29. Liu R, Li H, Liu L, Yu J, Ren X. Fibroblast activation protein A potential therapeutic target in cancer. Cancer Biology \& Therapy. 2012;13(3):pp.123-9.

30. Nasution N. Karakteristik Pasien Kanker Kolorektal di RSUP H. Adam Malik Medan 2015-2017. 2018. Available from : http://repositori.usu.ac.id/handle/123456789/11025

31. Arifputera A, Aninditha A, Tanto. Kapita Selekta Kedokteran. $4^{\text {th }}$ ed. Medical Aesculapius. Jakarta. 2014;pp.222-5.

32. Panduan Penatalaksanaan Kanker Kolorektal. 2017. Komite Penanggulangan Kanker Nasional. Kementerian Kesehatan Republik Indonesia. 2017.

33. Koelzer VH, Dawson H, Zlobec H, Lugli A. Colon: Colorectal Adenocarcinoma. In Atlas of Genetics and Cytogenetics in Oncology and Haematology. 2012.

34. da Silva RMS, Queiroga EM, Paz AR, Neves FFP, Cunha KS, Dias EP. Standardized Assessment of The Tumor-Stroma Ratio in Colorectal Cancer: Interobserver Validation and Reproducibility of a Potential Prognostic Factor. Clinical Pathology.2021;14:pp.1-4. 
35. American Cancer Society. Understanding Your Pathology eport: Invasive Adenocarcinoma of the Colon. 2017.

36. Sanberg TP, Oosting J, van Pelt GW, Mesker WE, Tollenaar RAEM, Morreau H. Molecular profiling of colorectal tumors stratified by the histological tumor-stroma ratio - Increased expression of galectin-1 in tumors with high stromal content. Oncotarget. 2018; 9(59), pp.31502-15.

37. Zhao K, Li Z, Yao S, Wang Y, Wu X, Xu Z, et al. Artificial intelligent quantified tumor-stroma ratio is an independent predictor for overall survival in resectable colorectal cancer. EBioMedicine. 2020:pp.19.

38. College of American Pathologist (CAP). Protocol for the Examination of Resection Specimens From Patients With Primary Carcinoma of the Colon and Rectum. 2020

39. Zhu Y, Jin Z, Qian Y, Shen Y, Wang Z. Prognostic value of Tumor-Stroma Ratio in Rectal Cancer: A Systematic Review and Meta-analysis. 2021; pp.1-9.

40. Wikberg ML, Edin S, Lunberg IV, van Guelpen, Dahlin AM, Rutegard J, et al. High intratumoral expression of fibroblast activation protein (FAP) in colon cancer is associated wiyh poorer patient prognosis. Tumor Biol. 2013: pp.1013-20.

41. Shi M, Yu DH, Chen Y, Chen YZ, Jing Z, Liu QH, et al. Expression of fibroblast activation protein in human pancreatic adenocarcinoma and its clinicopathological significance. World J Gastroenterol. 2012;18:pp.840-6.

42. Yuan Z, Hu H, Zhu Y, Zhang W, Fang Q, Qioau T, et al. Colorectal cancer cell intrinsic fibroblast activation protein alpha binds to Enolase1 and activates NF- $\kappa \mathrm{B}$ pathway to promote metastasis. Cell Death and Disease. 2021(12):pp.1-15.

43. Errarte P, Guarch R, Pulido R, Blanco L, Nunes-Xavier CE, Beitia M, et al. The Expression of Fibroblast Activation Protein in Clear Cell Renal Cell Carcinomas Is Associated with Synchronous Lymph Node Metastases. PLoS One. Public Library of Science.2016(11):pp.1-13.

44. Sahai E, Astsaturov I, Cukierman E, DeNardo DG, Egeblad M, Evans RM, et al. A framework for advancing our understanding of cancer-associated fibroblasts. Nat Rev Cancer. 2020;20(3): pp.174-86.

45. Lebleu VS, Kalluri R. A peek into cancer-associated fibroblasts : origins, functions and translational impact. Dis Model Mech. 2018;11: pp.1-9.

46. Hao Y, Baker D, Dijke PT. TGF- $\beta$-mediated epithelial-mesenchymal transition and cancer metastasis. International Journal of Molecular Sciences. 2019: pp.1-34. 